\title{
THE DIRICHLET PROBLEM FOR THE VIBRATING STRING EQUATION
}

\author{
D. G. BOURGIN AND R. DUFFIN
}

This note considers the Dirichlet and Neumann type boundary value problem for the simple vibrating string equation. The detailed study for a special boundary is timely in view of certain categorical statements in the recent literature. ${ }^{*}$ The results obtained below indicate how such statements are to be modified. $\dagger$ Of independent interest is the novel procedure, stemming from Lemma 1, for proving uniqueness in Theorems 1 and 2. The method is of wide utility and leads to interesting generalizations.

For convenience we use $t$ for $v \tau$, where $v$ and $\tau$ refer to the velocity of wave propagation and the time, respectively. The string equation is then

$$
L[y] \equiv y_{x x}-y_{t t}=0,
$$

and the data are given on the boundaries of the finite rectangle

$$
0 \leqq x \leqq S, \quad 0 \leqq t \leqq T .
$$

We denote the ratio $T / S$ by $\alpha$. The term "rectangle," used in the sequel, unless otherwise qualified, refers to the closed rectangle defined in (1.1).

We shall need the following lemma.

LEMMA 1. If $y(x, t)$ is continuous in both real arguments in the rectangle, and if $F(p, u)$ is defined as

$$
F(p, u)=\int_{0}^{T} \int_{0}^{S} e^{i(x p+u t)} y(x, t) d x d t,
$$

then $F(p, u)$ is an entire function in each of the complex variables $p$ and $u$.

* For example: "Dagegen würde ein Randwertproblem im Falle unserer hyperbolischen Differentialgleichung (our (1)) sinnlos sein," Courant-Hilbert, Methoden der Mathematischen Physik, vol. 2, p. 178. ". . . le problème de Dirichlet ne peut se poser pour le cas hyperbolique," J. Hadamard, L'Enseignement Mathematique, vol. 35 (1936), p. 26. Some of Hadamard's surmises are not borne out for the special situation we treat. Cf. pp. 26, 29 and notes on p. 29, loc. cit.

$\dagger$ In "principle," a physical realization of the Dirichlet problem is afforded by taking photographs of a vibrating string at two different times. However, "practically," our analysis is entirely ineffective, not for the reason of overdetermination (as in the illuminating instance on which Courant founds his mathematical conclusion) but because of the physical unpreciseness and inconstancy of the all important $\alpha$. 
The lemma is easily established even for less restricted $y(x, t) .^{*}$

THEOREM 1. Let $y(x, t)$ be a solution of (1) in the interior of the rectangle and let $y(x, t)$ vanish on the boundary. Furthermore, $y(x, t)$ is to be of class $C^{\prime} \dagger$ in the closed rectangle and $y_{x x}$ and $y_{t t}$ are to be Lebesgue summable over the rectangle. Then, in order that the solution $y(x, t)=0$ be unique, it is necessary and sufficient that $\alpha$ be an irrational number.

We take up the "sufficiency" aspect first. Our hypotheses justify the application of Green's theorem $\ddagger$ to (1) in the form

$$
\begin{aligned}
& \int_{0}^{T} \int_{0}^{S}\left\{e^{i(p x+u t)} L[y]-y L\left[e^{i(p x+u t)}\right]\right\} d x d t \\
&=\left(p^{2}-u^{2}\right) F(p, u)=\left.\int_{0}^{S}\left(e^{i(p x+u t)}\left(y_{t}(x, t)-i u y(x, t)\right)\right)\right|_{t=0} ^{t=T} d x \\
&-\left.\int_{0}^{T}\left(e^{i(p x+u t)}\left(y_{x}(x, t)-i p y(x, t)\right)\right)\right|_{x=0} ^{x=S} d t .
\end{aligned}
$$

On setting $y=0$ on the boundary, we obtain in the notation of (2)

$$
\begin{aligned}
& \left(p^{2}-u^{2}\right) F(p, u) \\
& =\left.\int_{0}^{S} e^{i(p x+u t)} y_{t}(x, t)\right|_{t=0} ^{t=T} d x-\left.\int_{0}^{T} e^{i(p x+u t)} y_{x}(x, t)\right|_{x=0} ^{x=S} d x .
\end{aligned}
$$

Since $F(p, u)$ is entire, according to Lemma (1), the left side of (3.1) vanishes for $p= \pm u$. Therefore

$$
\begin{aligned}
& \left.\int_{0}^{S}\left(e^{i u(t+x)} y_{t}(x, t)\right)\right|_{t=0} ^{t=T} d x-\left.\int_{0}^{T}\left(e^{i u(t+x)} y_{x}(x, t)\right)\right|_{x=0} ^{x=S} d t=0 \\
& \left.\int_{0}^{S}\left(e^{i u(t-x)} y_{t}(x, t)\right)\right|_{t=0} ^{t=T} d x-\left.\int_{0}^{T}\left(e^{i u(t-x)} y_{x}(x, t)\right)\right|_{x=0} ^{x=S} d t=0 .
\end{aligned}
$$

On subtracting these equations and taking the real part, there results (3.3) $\sin u T \int_{0}^{S} y_{t}(x, T) \sin u x d x-\sin u S \int_{0}^{T} y_{x}(S, t) \sin u t d t=0$.

In view of the incommensurability of $T$ and $S$, the special choices $u=n \pi / T$ and $u=n \pi / S$ imply

* Theorem 5.31, Whittaker and Watson, Modern Analysis, 3d edition, is the main tool.

$\dagger$ Class $C^{n}$ implies continuity in all arguments through derivatives of order $n$.

$\$$ The essentials of the argument are given in Horn, Partielle Differentialgleichungen, $2 \mathrm{~d}$ edition, p. 107. 


$$
\int_{0}^{T} y_{x}(S, t) \sin n \pi t / T d t=\int_{0}^{S} y_{t}(x, T) \sin n \pi x / S d x=0 .
$$

We may extend $y_{t}(x, T)$ to all values of $x$ by requiring it to be an odd periodic function of period $2 S$. This extended function is at worst piecewise continuous and summable and accordingly admits of a Fourier sine expansion, summable $C^{1}{ }^{*}$ According to (3.4) the Fourier coefficients vanish. The supposed continuity in the range $0 \leqq x \leqq S$ allows the deduction $y_{t}(x, T)=0$. $\dagger$ Similarly $y_{x}(S, t)=0$. The symmetry of the problem then guarantees the vanishing of $y_{t}(x, 0)$ and $y_{x}(0, t)$. It follows from (3.1) that

$$
F(p, u)=0
$$

if $p \neq \pm u$. This latter restriction may be waived, however. In fact, consider an arbitrary value of $u$ and denote it by $u_{0} . F\left(p, u_{0}\right)$ vanishes for all points in any open neighborhood of $p=u_{0}$ in the complex $p$ plane, except, possibly, for $p=u_{0}$. However, since $F\left(p, u_{0}\right)$ is analytic in $p$, it follows that $F\left(u_{0}, u_{0}\right)$ is also 0 . Similarly $F\left(-u_{0}, u_{0}\right)=0$. Hence the deduction (3.5) is valid for all finite $p$ and $u$. For our present purpose we may consider $y(x, t)$ to be 0 for all points outside the fundamental rectangle. Then $F(p, u)$ is an identically vanishing Fourier transform of $y(x, t)$ whence $y(x, t)=0 . \S$

The necessity is easily verified. Indeed if $\alpha$ is rational we have the nonidentically vanishing solution

$$
y(x, t)=\sin n \pi x / S \sin n \pi t / S,
$$

where $n$ and $n \alpha$ are integers.

For the case envisaged in Theorem 1 it is worthwhile to remark an alternative proof which, though not directly capable of extension to a more general equation, may clarify the physical situation. Our starting point is the classic solution of (1), namely

$$
2 y(x, t)=y(x+t, 0)+y(x-t, 0)+g(x+t)-g(x-t),
$$

* E. C. Titchmarsh, Theory of Functions, pp. 414 and 426.

$\dagger$ Since we now have established vanishing Cauchy data on the upper side of the rectangle we can appeal to the theory of the so-called "mixed" problem to gain the conclusion in our theorem. However, it is of interest to carry through the method predicated on Lemma 1 to show its power and completeness.

$\ddagger$ Alternatively we may take the real part of the difference of equations (3.2) and (3.21), and also the imaginary part of their sum. With the justifiable definition of $y_{t}(x, 0)$ and $y_{x}(0, t)$ as odd periodic functions of $x$ and $t$, with periods $2 S$ and $2 T$ respectively, one then easily derives $y_{t}(x, 0)=y_{x}(0, t)=0$ as a consequence of $y_{t}(x, T)$ $=y_{x}(S, t)=0$.

$\S$ S. Bochner, Vorlesungen über Fourier Integralen, p. 192. 
where

$$
g(z)=\int_{0}^{z} y_{t}(x, 0) d x
$$

Now $y_{t}(x, 0)$ is of class $C^{0}$ and may be considered odd and periodic on the $x$ axis with period $2 S$. Since $g(z)$ is the integral of an odd periodic function it is itself periodic with the same period $2 S$.

The identical vanishing of $y$ on the boundary implies

$$
0=g(x+T)-g(x-T) .
$$

Thus $g(z)$ has a second period, $2 T$. Accordingly

$$
g(2 n T+2 m S)=g(0)
$$

for all integer values of $|n|$ and $|m|$. Since $T$ and $S$ are incommensurable it follows that, for arbitrary $x$ and $\epsilon$, positive or negative integers $\bar{n}$ and $\bar{m}$ may be found such that

$$
|x+2 \bar{n} T+2 \bar{m} S|<\epsilon .
$$

Relation (4.3) is, in fact, an immediate consequence of the well known Kronecker inequality. Thus the points $\{2 n T+2 S m\}$ are everywhere dense on the interval

$$
-S-T \leqq z \leqq S+T .
$$

Manifestly $g(z)$ is continuous on the interval defined in (4.4). Since a continuous function on an interval is determined uniquely by its values on a dense set of points, it follows from (4.2) that

$$
g(z)=g(0)
$$

for all $z$ in the interval in question. It is now seen that (4) guarantees $y(x, t)=0$.

We may also establish uniqueness in the case of the Neumann problem (or more generally, for the case that Dirichlet data are given on some sides and Neumann data on the others). For instance we have the following result.

THEOREM 2. Let $y(x, t)$ be a solution of (1) for interior points and be of class $C^{1}$ in the rectangle, with $y_{x x}$ and $y_{t t}$ summable over the rectangle. Furthermore, let $y_{t}$ and $y_{x}$ vanish on the horizontal and vertical sides of the boundary, respectively. Then irrationality of $\alpha$ is the necessary and sufficient condition that the only solution satisfying our hypotheses be a constant.

The necessity follows from the observation that, for rational $\alpha$, 


$$
y(x, t)=\cos n \pi x / S \cos n \pi t / S
$$

is a solution for values of $n$ such that $n \alpha$ is also an integer.

The demonstration of sufficiency requires, merely, obvious modification of the analysis in our first method of proof of Theorem 1. Thus, on setting $p= \pm u$ in (3) we obtain as the analogues of relations. (3.2) and (3.21),

$$
\begin{aligned}
& \left.\int_{0}^{S} e^{i u(x+t)} y(x, t)\right|_{t=0} ^{t=T} d x=\left.\int_{0}^{T} e^{i u(x+t)} y(x, t)\right|_{x=0} ^{x=S} d t, \\
& \left.\int_{0}^{S} e^{i u(x-t)} y(x, t)\right|_{t=0} ^{t=T} d x=\left.\int_{0}^{T} e^{i u(x-t)} y(x, t)\right|_{x=0} ^{x=S} d t .
\end{aligned}
$$

Manifestly we may take $y(x, T), y(x, 0), y(S, t), y(0, t)$ as even periodic functions of periods $2 S$ and $2 T$ according as the variable argument is $x$ or $t$. Formulas (5.1) and (5.11) imply

$$
\sin u T \int_{0}^{S} \cos u x y(x, T) d x=\sin u S \int_{0}^{T} \cos u t y(S, t) d t .
$$

The choices $u=n \pi / S$ and $u=n \pi / T$ show that the integrals on either side vanish for all positive integers $n$ and are unrestricted for $n=0$. We may paraphrase an earlier argument to deduce that $y(x, t)$ is a constant $K$ on the boundary. On evaluation of the boundary integrals, (3) now yields

$$
F(p, u)=K\left(e^{i u^{T}}-1\right)\left(e^{i p s}-1\right) / u p .
$$

This is the transform of a function vanishing outside the rectangle. Inside and on the boundary

$$
y(x, t)=K \text {. }
$$

The restriction that $y$ be of class $C^{1}$, coupled with the almost everywhere equivalence of two Lebesgue summable functions having the same transform, implies that (5.4) is unique.

THEOREM 3. Let $y(x, t)$ satisfy all the conditions imposed in Theorem 1 save that the boundary values are now arbitrary functions of class $C^{1}$ which vanish at the four corners. Then if $\alpha$ is irrational, a solution $y(x, t)$, if it exists, is unique.

The demonstration is along conventional lines. Suppose $y_{1}$ and $y_{2}$ are distinct solutions. Define $y^{*}$ by $y^{*}=y_{1}-y_{2}$. Clearly $y^{*}$ satisfies the hypotheses of Theorem 1 whence it follows that $y_{1}$ and $y_{2}$ are identical. 
The ratio $\alpha$ plays a determining role in the demonstration of actual existence of solutions. Our attention will be focussed on $\alpha$ values satisfying the inequality $(\mathrm{E})$

$$
|\alpha-m / n|>A / n^{K+1},
$$

where $A$ and $K$ are fixed, $m, n, K$ are positive integers and $m \leqq 2 \alpha n$. The algebraic numbers of degree $K+1$ satisfy (E). Moreover the set of $\alpha$ values which do not satisfy (E) for any choice of $K$ is known to be of linear Lebesgue measure zero.*

Lemma 2. If $\alpha$ satisfies (E), then for each integer $N$, sufficiently large, there is associated an integer $M^{\prime}$, depending on $N$, such that $1 / 2 N>\left|1 / \alpha-M^{\prime} / N\right|>B / N^{K+1}$.

We replace $n$ by $M$ and $m$ by $N$ in (E). Clearly we may select a "best" $M$, denoted by $M^{\prime}$, for each $N$ in the sense

$$
|\alpha-N / M| \geqq\left|\alpha-N / M^{\prime}\right|, \quad M \neq M^{\prime} .
$$

For $N_{0}$ sufficiently large and fixed

$$
2 \alpha>N / M^{\prime}>\alpha / 2,
$$$$
N>N_{0} \text {. }
$$

Hence by (E) and the defining relation (6) for $M^{\prime}$, we plainly have

$$
\begin{aligned}
1 / 2 N \geqq\left|1 / \alpha-M^{\prime} / N\right|=\left|\alpha-N / M^{\prime}\right| M^{\prime} / \alpha N \\
\geqq \frac{A}{\alpha}\left(\frac{N}{M^{\prime}}\right)^{K} / N^{K+1}=B / N^{K+1}, \quad N>N_{0},
\end{aligned}
$$

where $B=(A / \alpha)(\alpha / 2)^{K}$.

The assigned boundary functions $y(x, 0), y(x, T)$ on the two horizontal sides of the rectangle of (1) may be extended to all $x$ values by requiring the extended functions to be odd and periodic of period $2 S$. Similarly the extended functions $y(0, t), y(S, t)$ are odd and periodic of period $2 T$. It is in this sense that subsequent references to "extended boundary functions" are to be understood.

ThEOREM 4. If (a) $\alpha$ satisfies (E) for a fixed $K$ and if (b) the four extended boundary functions are of class $\dagger C^{4+K}$ for $-S \leqq x \leqq S$ or $-T \leqq t \leqq T$, then there exists a unique solution $y(x, t)$ of $(1)$, satisfying the boundary conditions, such that (c) $y_{x x}$ and $y_{t t}$ are summable over the rectangle and (d) $y$ is of class $C^{1}$ in the rectangle. This solution is actually of class $C^{2}$ in the rectangle.

* E. Borel, Theorie des Fonctions, 3d edition, p. 27.

$\dagger$ The condition on the $(K+4)$ th derivatives may evidently be weakened. 
Condition (b) implies that the boundary functions may be expanded in Fourier sine series. Furthermore

$$
a_{n}, A_{n}, b_{n}, B_{n}=o\left(n^{-4-K}\right), *
$$

where the constants on the left side of (7) are the $n$th coefficients in the sine series expansions of $y(x, 0), y(x, T), y(0, t)$ and $y(S, t)$, respectively. For any $n$ we may find $m^{\prime}$ depending on $n$, such that

$$
\left|\alpha n-m^{\prime}\right|<1 / 2 \text {. }
$$

Then for $n \geqq n_{0} \geqq \max \left(3, A^{1 / K}\right)$,

$$
|\sin n \pi \alpha|=\left|\sin \pi\left(n \alpha-m^{\prime}\right)\right| \geqq\left|\sin \pi A / n^{K}\right| \geqq A / 4 n^{K} .
$$

In view of Lemma 2 we may establish in a similar way

$$
|\sin n \pi / \alpha| \geqq B / 4 n^{K}, \quad n \geqq n_{1} .
$$

We assert that a solution satisfying the conditions of our theorem is given by

$$
\begin{aligned}
y(x, t)= & \sum\left\{\frac{a_{n} \sin (n \pi(T-t) / S)+A_{n} \sin n \pi t / S}{\sin n \pi \alpha} \sin n \pi x / S\right. \\
& \left.+\frac{b_{n} \sin (n \pi(S-x) / T)+B_{n} \sin (n \pi x / T)}{\sin n \pi / \alpha} \sin n \pi t / T\right\} .
\end{aligned}
$$

The right side of (7.4) formally satisfies (1). In view of (7), (7.2), and (7.3) it is clear that

$$
\left|\frac{a_{n}}{\sin n \pi \alpha}\right|, \cdots,\left|\frac{B_{n}}{\sin n \pi / \alpha}\right|=o\left(n^{-4}\right) .
$$

This implies that the right side of (7.4) is the sum of four uniformly convergent Fourier series and these series remain uniformly convergent on differentiation twice by either $x$ or $t$. Accordingly, the four formal series on the right side of (7.4) are Fourier series defining functions certainly of class $C^{2}$ in the rectangle. Thus $y_{x x}, y_{t t}$ are summable. Finally, no other solution of our problem of class $C^{1}$ with summable $y_{x x}$ and $y_{t t}$ in the rectangle can exist, according to Theorem 2.

Incidentally, the form of (7.4) indicates that if $\alpha$ is rational, then a necessary condition for the existence of a (non-unique) solution is that $A_{\nu}=a_{\nu} \cos \nu \pi \alpha, B_{\mu}=b_{\mu} \cos \mu \pi / \alpha$, where $\nu$ and $\mu$ are integers such that $\nu \alpha$ and $\mu / \alpha$ are also integers.

Hypothesis (b) of Theorem 4 is a sufficient but by no means necessary condition. Consider for instance, for $\alpha<1$,

* Titchmarsh, loc. cit. 
(8) $\begin{aligned} y(x, t) & =x(T-S-t)\left(x^{2}+(T-t)^{2}-2 S(T-t)\right) ; 0 \leqq x \leqq T-t, \\ & =(x-S)(T-t)\left(x^{2}+(T-t)^{2}-2 S x\right) ; T-t \leqq x \leqq S .\end{aligned}$

Independently of the value of $\alpha, y(x, 0)$ is merely of class $C^{2}$, yet $y(x, t)$ is a solution of class $C^{2}$.

Nevertheless some such hypothesis as (b) must be admitted to guarantee existence of the required solution in all cases. We take $A=1$ and denote by $K^{\prime}+1$ the smallest integer $K$ for which $\alpha$ satisfies (E) except, possibly, for a finite number of values of $n$. We shall suppose $K^{\prime} \geqq 3$ in the sequel. Accordingly there exists an infinite number of pairs of values of $n$ and $m$, denoted by $n_{i}$ and $m_{i}$, such that

$$
\left|\alpha-m_{i} / n_{i}\right|<1 / n_{i}^{K^{\prime}+1} \text {. }
$$

Hence for $n_{i}>3$

$$
\left|\sin n_{i} \pi \alpha\right| \leqq \pi / n_{i}{ }^{K^{\prime}},\left|\cot n_{i} \pi \alpha\right| \geqq n_{i}{ }^{K^{\prime}} / 2 \pi \text {. }
$$

We take Dirichlet data vanishing except on the base and the following coefficients, $a_{n}$, in the Fourier sine expansion of $y(x, 0)$ :

$$
a_{n_{i}}=1 / n_{i} K^{\prime+1}, \quad a_{n}=0, \quad n \neq n_{i} .
$$

Thus the extended $y(x, 0)$ is certainly of class $C^{K^{\prime}-1}$. Suppose a solution $y(x, t)$ of class $C^{2}$ exists. It is easily shown then by an argument based on (4) for instance, if we bear in mind the prescribed vanishing of $y(x, t)$ on the vertical sides, that $y_{t}(x, 0)$ may be extended to an odd periodic function of class $C^{0}$. Moreover $y_{t}(x, 0)$ is of class $C^{1}$ on the base of the rectangle. Accordingly $y(x, t)$ and $y_{t}(x, 0)$ are expansible in absolutely and uniformly convergent Fourier series lacking the terms $\cos \pi n x / S$. The nonzero Fourier coefficients, $a_{n}^{\prime}$ for $y_{t}(x, 0)$ must be

$$
a_{n_{i}}^{\prime}=-\pi\left(\cot n_{i} \pi \alpha\right) / S n_{i}^{K^{\prime}}
$$

In view of (8.2)

$$
\left|a_{n_{i}}^{\prime}\right|>1 / 2 S \text {. }
$$

This contradicts the deduction that the $\left\{a_{n}^{\prime}\right\}$ are Fourier coefficients. Hence $y(x, t)$ cannot be of class $C^{2}$ in the rectangle. Therefore hypothesis (b) cannot be weakened to admit data of continuity class inferior to $C^{K^{\prime}}$ and, indeed, if $K^{\prime} \geqq 4$ an obvious extension shows that the lowest class is $C^{K^{\prime}+1}$ at least.

UNIVERSITY OF ILLINOIS AND PURdue UNIVERSITY 